\title{
565 COMBINATORIAL HSCS AND ANTI-PD-1 THERAPY IN MICROSATELLITE STABLE COLORECTAL CANCER
}

Bayli DiVita Dean*, John Figg, Laura Falceto Font, Connor Francis, Duane Mitchell, Catherine Flores. University of Florida, Gainesville, FL, USA

Background Colon cancer (CRC) is the second leading cause of cancer-related deaths in the US. CRC incidence is on the rise and there is an alarming increase in young onset CRC cases. Immune checkpoint inhibitors (ICIs) have yielded promising anti-tumor results in microsatellite instable high (MSIhigh) patients, which represent only $15 \%$ of tumors. The remaining $85 \%$ are denoted as microsatellite stable (MSS) and are unresponsive to ICI. Using a murine glioma model, our group has previously found the combination of anti-PD-1 and a transfer of hematopoietic stem cells (HSCs) can sensitize mice that are resistant to anti-PD-1 alone. We evaluated survival after treatment with this combinatorial platform 3 or 5 days post-implantation in subcutaneous CRC-bearing mice and also phenotyped the splenic compartment of mice at endpoint. Methods 1x106 MSS CRC cells, CT26, were subcutaneously injected into the right flank of $\mathrm{BALB} / \mathrm{cJ}$ mice. 3 or 5 days later, HSCs were isolated from naïve $\mathrm{BALB} / \mathrm{cJ}$ mice and injected through the tail vein into CT26-bearing mice and were also given $10 \mathrm{mg} / \mathrm{kg}$ anti-PD-1. Mice were given 3 additional doses of anti-PD-1 for a total of doses either every 3 or 5 days. Mice were sacrificed when tumors reached $1.5 \mathrm{~cm}$ at its widest point and spleens were excised and stained for flow cytometry.

Results When mice were treated with HSC/anti-PD-1 3 days post-tumor implantation, we observed a statistically significant increase in survival in mice that received combinatorial HSCs and anti-PD-1 relative to no treatment control mice ( $p=0.0034$, Mantel-Cox long-rank test) as well as mice that received HSCs alone $(p=0.0462$, Mantel-Cox log-rank test. In the same 3 day cohort, no differences in the frequency of $\mathrm{T}$ cell populations were observed. However, we found mice that received this combination therapy had a significant increase in the frequency of splenic CD11c+ MHC II+ dendritic cells (DCs) relative to no treatment control mice $(p=0.0364$, Mann-Whitney $\mathrm{t}$ test). When mice were treated with HSC/ anti-PD-1 5 days post-tumor implantation, we found a statistically significant increase in survival of mice treated with combinatorial HSCs and anti-PD-1 compared to no treatment control mice ( $p=0.0024$, Mantel-Cox log-rank test) and relative to mice that received HSC monotherapy $(p=0.0462$, Mantel-Cox log-rank test).

Conclusions These results suggest combinatorial HSCs and anti-PD-1 represents a promising therapeutic axis in a murine model of MSS CRC. In addition, the increase in splenic DCs suggests the mechanism behind this anti-tumor response may be expansion of DCs within the periphery.

Ethics Approval All animal work approved through University of Florida IACUC \# 201910777

http://dx.doi.org/10.1136/jitc-2021-SITC2021.565 